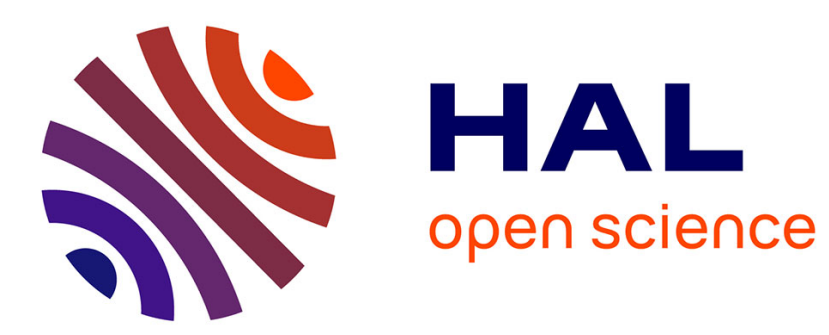

\title{
ADSORBED SOLID XENON AND KRYPTON ON GRAPHITE-DIFFRACTION STUDIES AND MODEL CALCULATIONS
}

J. Venables, P. Schabes-Retchkiman

\section{- To cite this version:}

J. Venables, P. Schabes-Retchkiman. ADSORBED SOLID XENON AND KRYPTON ON GRAPHITE-DIFFRACTION STUDIES AND MODEL CALCULATIONS. Journal de Physique Colloques, 1977, 38 (C4), pp.C4-105-C4-114. 10.1051/jphyscol:1977416 . jpa-00217132

HAL Id: jpa-00217132

https://hal.science/jpa-00217132

Submitted on 1 Jan 1977

HAL is a multi-disciplinary open access archive for the deposit and dissemination of scientific research documents, whether they are published or not. The documents may come from teaching and research institutions in France or abroad, or from public or private research centers.
L'archive ouverte pluridisciplinaire HAL, est destinée au dépôt et à la diffusion de documents scientifiques de niveau recherche, publiés ou non, émanant des établissements d'enseignement et de recherche français ou étrangers, des laboratoires publics ou privés. 


\title{
ADSORBED SOLID XENON AND KRYPTON ON GRAPHITE-DIFFRACTION STUDIES AND MODEL CALCULATIONS
}

\author{
J. A. VENABLES and P. S. SCHABES-RETCHKIMAN \\ School of Mathematical and Physical Sciences, \\ University of Sussex, Brighton, BN1 9QH, Sussex, England
}

\begin{abstract}
Résumé. - On souligne ici l'importance des dernières études de diffraction qui ont permis d'établir les diagrammes de phase et les isostères des couches monoatomiques de Kr et Xe solides adsorbés sur le graphite. La mesure des paramètres réticulaires permet d'obtenir des isostères avec une précision de $\sim 0,6 \%$ qui peuvent être utilisés pour tester des modèles approfondis de mécanique statistique de ces couches solides. Ces modèles utilisent les potentiels de gaz rares les plus précis dans le cadre du formalisme de la dynamique du réseau. On s'applique à étudier les transitions solide-gaz et solide-solide (localisé-délocalisé). On montre que (i) l'énergie à trois corps de Sinanoglu-Pitzer est significative mais approximativement 0,4 fois plus grande que celle proposée originalement, (ii) les dislocations interfaciales modifient la forme de la transition solide-solide ; de plus l'énergie de dislocation de Frank-Van der Merwe permet de rendre compte convenablement de la transition du second ordre observée, et (iii) les dislocations modifient considérablement les intensités diffractées.
\end{abstract}

\begin{abstract}
The importance of recent diffraction studies in establishing phase boundaries and isosteres for solid adsorbed monolayers of $\mathrm{Kr}$ and $\mathrm{Xe}$ on graphite is emphasised. Lattice parameter measurements give isosteres to $\sim 0.6 \%$ accuracy which can be used to test detailed statistical mechanical models of the solid layers. These models are developed using the most accurate rare gas potentials in conjunction with the cell model of the lattice dynamics. By concentrating on the solid-gas and solid-solid (registry-disregistry or localised-unlocalised) phase transitions, we show that: (i) the Sinanoglu-Pitzer (substrate-mediated) three-body energy is significant but approximately 0.4 times as large as originally proposed; (ii) that misfit dislocations modify the form of the solid-solid phase transition, and that the Frank-van der Merwe form of the dislocation energy can be made to fit the observed (second-order) transitions reasonably well ; and (iii) that dislocations also modify diffraction intensities considerably.
\end{abstract}

1. Introduction. - It is clear from this conference that the physics of quasi-two-dimensional (2D) systems is arousing much current interest. The physisorption of rare gases on graphite has long been studied and is of especial interest, since one can begin to model the interactions between the rare gas atoms, and between the rare gas atoms and the -graphite, with some precision. These interactions, when coupled with a suitable description of the motion of the adatoms and the substrate atoms, form the basis for evaluating detailed statistical mechanical models of the adsorbed state and for testing them against experiment. This approach has been reviewed in a recent book [1].

The gases $\mathrm{Kr}$ and $\mathrm{Xe}$ are interesting for several reasons. Firstly, they are the most classical of the rare gases because of their large masses, so that classical theories can be used for the most part. Secondly, much data has recently been acquired using volumetric analysis $[2,3,4,5]$, Auger electron spectroscopy $[6,7,8]$ and ellipsometry [9] and various diffraction techniques discussed in this paper. As a result of this work, the 2 D-phase diagrams of $\mathrm{Kr}$ and $\mathrm{Xe}$ on graphite are becoming known, and exhibit a considerable richness of structure. The diagrams are shown in figures 1 and 2 as a function of the thermodynamic variables $\log p$ (torr) versus $1 / T$. It is by no means clear that all the phases which exist in equilibrium have yet been discovered.

These phase diagrams show phase equilibrium lines, for example between a $2 \mathrm{D}$ solid and a $2 \mathrm{D}$ gas, and the slope of such lines can be calculated for a given model. A model will however give more than this : it will also give the lines of constant density of atoms $n(p, T)$, whose slope on a $(\ln p-1 / T)$ plot is the isosteric heat of adsorption, $q_{\mathrm{st}}$. Thus to test the models we really need to measure $n(p, T)$ accurately as well as determine the phase equilibrium lines. We also need to do accurate calculations for realistic models of the adsorbed state. This is most feasible for the solid $2 \mathrm{D}$ phases and for the adsorbed gas. For the solid phases we can aim to combine accurate interatomic potentials 


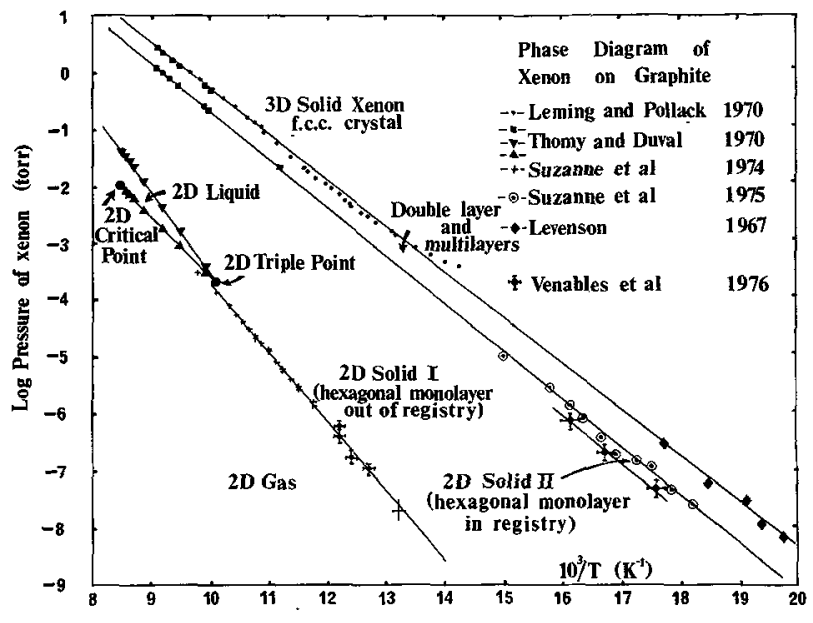

FIG. 1. - Phase diagram of Xe on graphite.

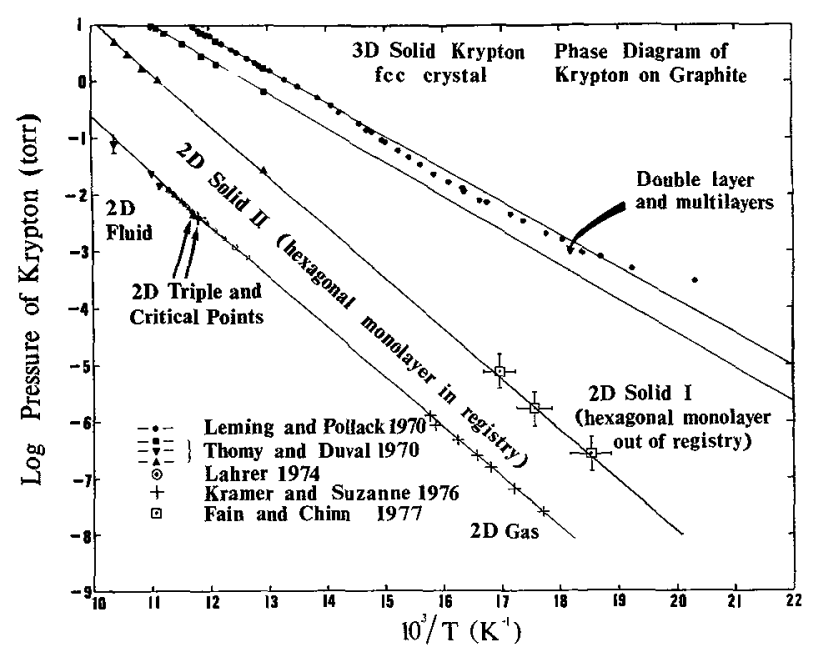

FlG. 2. - Phase diagram of Kr on graphite.

with an adequate description of the lattice dynamics to explain the thermodynamics, in the same way as has been done recently for bulk ( $3 \mathrm{D})$ rare gas solids [10].

In this paper we concentrate on the use of diffraction experiments to give information about the structure of the solid layers, and to measure $n(p, T)$ accurately. We then discuss models of the layers and discuss the solid-gas and solid-solid phase transitions using recent interatomic potentials for $\mathrm{Kr}$ and $\mathrm{Xe}$.

2. Diffraction techniques and results. 2.1 TECHNIQUES. - Diffraction techniques can measure both the orientational order (epitaxial orientation) of a solid adsorbed layer and its lattice parameter. As with the other techniques, there is a distinction between those techniques which can be used on a single exposed substrate surface and those which require a large volume of adsorbate, which have to use exfoliated graphite (e.g., grafoil). As has been pointed out recently [11] this distinction must always be borne in mind, as there is often a considerable amount of adsorbate condensed on the re-entrant corners of the exfoliated material, and that this may lead to adsorption isotherms which are not as sharply stepped as on a single surface $[8,11]$.

Neutron diffraction, since it is not surface sensitive, has been done on exfoliated graphite for $\mathrm{N}_{2}$ [12] and $\mathrm{O}_{2}$ [13], very recently for $\mathrm{Kr}$ [38] but not for Xe. Low energy electron diffraction (LEED) [6, $7,14,15]$ and transmission high energy electron diffraction (THEED) [16] have been done on single exposed surfaces of graphite, in the latter case in the form of thin ( $<10 \mathrm{~nm}$ thick) plates. Both of these techniques can detect a single solid adsorbed monolayer. It is more difficult to measure the lattice parameter of the layer accurately. Early LEED work on xenon $[6,14]$ indicated that the gas condensed into the so-called $\sqrt{3} \times \sqrt{3}$ structure, with the gas atoms occupying the centre of every third graphite hexagon. However, more recent THEED work [16] has shown that under these conditions the lattice parameter is not the same as the relevant graphite distance, but is larger by about $6 \%$. This implies that most current LEED apparati are not capable of resolving lattice parameter differences of this order. The reason for this is the lack of spatial coherence of the LEED beam which results in very broad spots. An improved apparatus [15] has given very encouraging results for $\mathrm{Kr}$, and now both LEED and THEED can measure the lattice parameter of an adsorbed solid monolayer to about $0.3 \%$ accuracy. An accuracy of order $0.3 \%$ has been claimed for neutron diffraction on quasipoly-crystalline samples of grafoil [12].

The lattice parameter of the adsorbed layer is an important thermodynamic parameter, as under certain conditions discussed below, it enables us to determine $n(p, T)$ and hence is a useful check on a model of the layer. The main condition is that the layer should not be rough; i.e. the number of vacancies and adatoms should be small. This is undoubtedly the case for $\mathrm{Kr}$ and Xe monolayers on graphite where independent estimates $[11,17]$ show the calculated vacancy concentration to be $<10^{-4}$ at temperatures $\leqslant 90 \mathrm{~K}$. Thus when there is a single adsorbed solid phase on the surface, a measurement of the lattice parameter to $0.3 \%$ accuracy determines $n(p, T)$ to $0.6 \%$. Ónly once lattice parameters become available at around $0.005 \%$ accuracy will we have to worry about vacancy effects in these systems.

Even at the present level of accuracy, the diffraction information gives probably the most accurate data for $n(p, T)$ for solid layers. Furthermore it is internally calibrated (by reference to the graphite lattice), so we do not have the problem of defining what is meant by monolayer 
coverage which is implicit in volumetric analysis, AES and ellipsometry. It is also rather insensitive to adsorption at special sites such as ledges, which is seen, for example, by AES [8]. Hence the diffraction data may conform more closely to the idealised model than other results. As against these advantages it is clear that the diffraction information is only useful from solid layers, and that the lattice parameter cannot be used to follow the progress of first order phase transitions such as the gas-solid transition.

Extra information may be obtained from diffraction intensities, and in a linear model one would expect the integrated intensity of a diffraction spot to be proportional to $n(p, T)$. This parameter has not yet been used to follow the progress of a phase transition, though there are indications that intensities vary during transitions [7] and that the intensities might be used to indicate the microstructure of the layer [16]. Preliminary results are discussed in the next section.

2.2 RESULTS. - Lattice parameters obtained by THEED for $\mathrm{Xe}$ [16] and by LEED for $\mathrm{Kr}$ [15] are shown in figures 3 and 4 . In the $X e$ case the

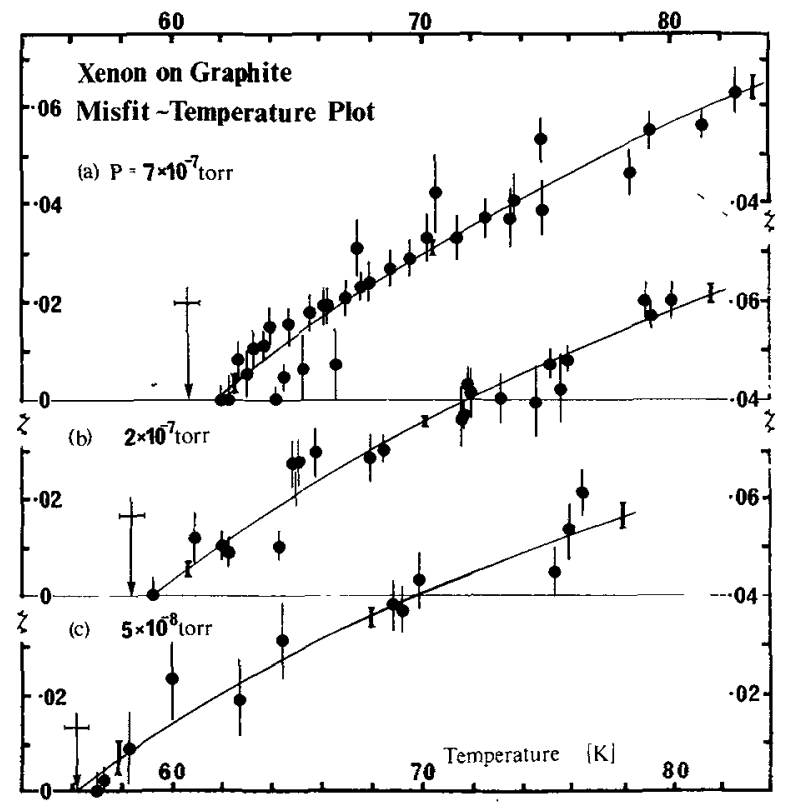

FrG. 3. - Lattice parameter measurements for Xenon on graphite by THEED [16].

temperature was varied at constant pressure and for $\mathrm{Kr}$ the pressure was varied at constant temperature. The regions of the phase diagram explored by these techniques ( $p \leqslant 10^{-4}$ torr) can be seen in figures 1 and 2 . In figures 3 and 4 , the misfit $m$ is related to $n$ by $m=n^{-1 / 2}-1$. For Xe, $m \geq 0$ and $n \leq 1, n$ decreasing as one goes towards the gas-solid equilibrium line. For $\mathrm{Kr} m \leq 0$ and $n \geq 1, n$ being equal to 1 at the gas-solid line and staying at 1 until a solid-solid transition occurs to a higher density solid.

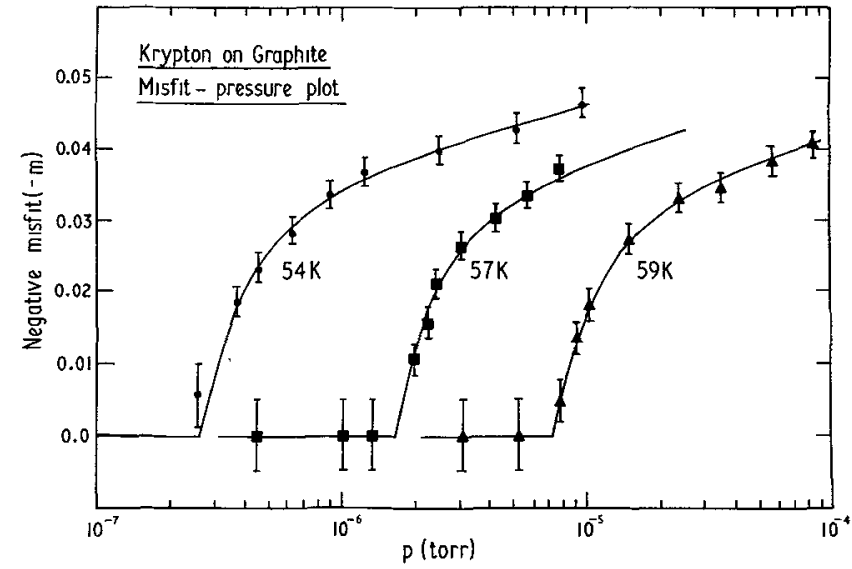

FIG. 4. - Lattice parameter measurements for Krypton on graphite by LEED ([15], and M. D. Chinn and S. C. Fain, Jr., these proceedings).

In the xenon case, the transition to the $n=1$ $(m=0)$ in-registry or localised state occurs at low temperature, but this transition occurs very close to the monolayer-double layer transition, so that few examples of this state have been found so far. The $\mathrm{Kr}$ solid-solid transition had previously been found at much higher pressures, by volumetric analysis [3]; the xenon transition is not yet well characterised. The shape of the curves in figures 3 and 4 indicate that these solid-solid transitions are of second order.

The intensities of diffraction spots also contain information, and the method of measurements of the diffracted intensity $I_{\mathrm{g}}$ and the incident electron beam intensity $I_{0}$ in THEED have been discussed previously [16]. More recently we have shown that the ratio $\left(I_{g} / I_{0}\right)$ can be measured with a precision of about $\pm 10 \%$ using a computer-controlled densitometer. The densitometer scans a $30 \times 30$ raster over the diffraction spot $g$ and the computer then calibrates these 900 intensity measurements, performs a sloping background subtraction and then fits the spot to a two-dimensional gaussian and finds the integrated intensity. Having previously scanned a plate which measured the incident intensity, we then obtained the ratio $\left(I_{\mathrm{g}} / I_{0}\right)$. The shape of the spot, the (very good) fit to the gaussian and the ratio $\left(I_{g} / I_{0}\right)$ are shown in figure 5.

As a result of these measurements we found that for $g=\{10\} I_{\mathrm{g}} / I_{0}=1.4 \pm 0.15 \times 10^{-4}$ and for $g=\{20\}, \quad I_{g} / I_{0}=3.3 \pm 0.3 \times 10^{-5}$, for xenon at $T=67.1 \mathrm{~K} p=4.7 \times 10^{-7}$ torr. Further experiments to search for systematic $p$ and $T$ dependencies are continuing. These intensity ratios are considerably less than the values $4.7 \pm 0.6 \times 10^{-4}$ and $1.48 \pm 0.2 \times 10^{-4}$ which would be expected for an undistorted (static) hexagonal monolayer. In the example quoted, the ratios are only $0.29 \pm 0.05$ and $0.22 \pm 0.04$ of that expected.

This discrepancy is too large to be accounted for 


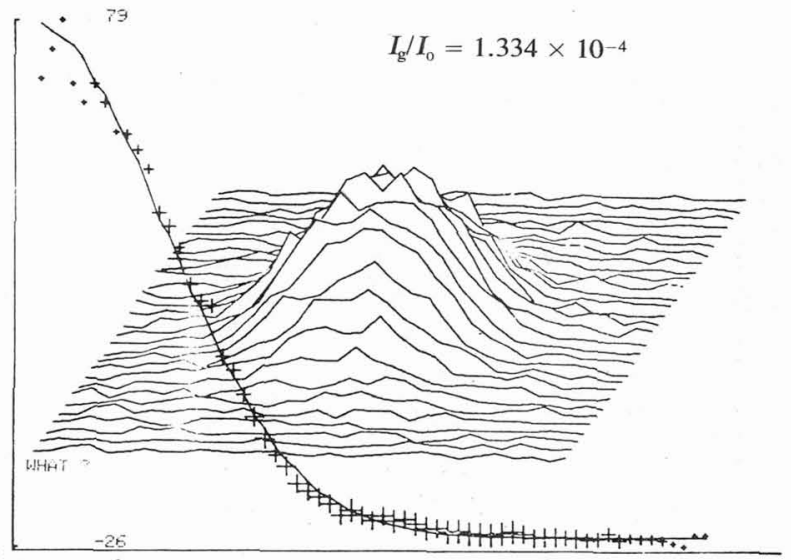

FIG. 5. - Intensity measurement of diffraction spots in THEED by computer controlled densitometer. The Gaussian fit (curve), the shape of the spot, and ratio $I_{\mathrm{g}} / I_{0}=1.334 \times 10^{-4}$ are displayed.

by a thermal Debye-Waller factor and we suggest in $\$ 4.3$ that these values are reasonable for a model of the adsorbed layer which contains an array of misfit dislocations. Since these same dislocations also explain the epitaxial orientation of the adsorbed solid, and can lead to a second order solid-solid phase transition $(\$ 3.3)$ it is clear that the model of a monolayer with misfit dislocations is quite close to the experimental facts for $\mathrm{Kr}$ and $\mathrm{Xe}$, and may be generalisable to other systems as well. Using this model we can also examine the gas-solid phase transition, and say something about the magnitude of many-body forces between adsorbed atoms. But first we discuss briefly the general method of modelling monolayers and their phase transitions, and outline what we know about interatomic interactions in rare-gas graphite systems.

3. Modelling the adsorbed state. 3.1 THERMODYNAMICS AND A STATISTICALMECHANICAL MODEL. - The thermodynamics of $2 \mathrm{D}$ adsorbed phases in equilibrium appear in several references e.g. $[1,19,20]$, but the account on which this description is based is that by Price and Venables (hereafter PV) [17]. For equilibrium between two adsorbed phases 1 and 2, and the gas phase $\mathrm{g}$, we must have the chemical potentials and spreading (2D) pressures equal, viz :

$$
\mu_{1}=\mu_{2}=\mu_{\mathrm{g}} \text { and } \phi_{1}=\phi_{2} .
$$

A phase equilibrium (or transition) line on a $p-T$ diagram is defined by

$$
\mathrm{d} \mu_{1}=\mathrm{d} \mu_{2}=\mathrm{d} \mu_{\mathrm{g}} .
$$

By expressing $\mu_{x}=h_{x}-T s_{x}$ and

$$
\mu_{\mathrm{g}}=h_{\mathrm{g}}-T \sigma+k T \ln p
$$

where $h_{x}, h_{\mathrm{g}}$ are enthalpies and $s_{x}, \sigma$ are entropies, the form of transition lines and isosteres can be deduced to be

$$
\ln p=' x / T+b
$$

where $a$ and $b$ are roughly temperature independent. Thus plots of $\log p-1 / T$ such as figures 1 and 2 are very convenient.

In the case of a transition line between two adsorbed phases :

$$
\begin{gathered}
a=\left[-h_{\mathrm{g}}+\left(n_{2} h_{2}-n_{1} h_{1}\right) /\left(n_{2}-n_{1}\right)\right] / k, \\
b=\left[\sigma-\left(n_{2} s_{2}-n_{1} s_{1}\right) /\left(n_{2}-n_{1}\right)\right] / k .
\end{gathered}
$$

For the adsorbed gas-solid transition it is usual simply to neglect $n_{1}$ in equation (4). For isosteres (lines of constant $n$ ) the finite difference terms in (4) are replaced by a differential, viz: $\partial\left(n_{x} h_{x}\right) / \partial n_{x}$ the isoteric heat of adsorption $q_{s t}$ is given by

$$
-q_{\mathrm{st}}=k\left(\frac{\mathrm{d} \ln p}{\mathrm{~d}(1 / T)}\right)_{n}=-h_{\mathrm{g}}+\frac{\partial(n h)}{\partial n} .
$$

The evaluation of these quantities is made by constructing a detailed statistical mechanical model for the partition function $Q$, and using the standard equation $F=-k T \ln Q$, with $\mu=(\partial F / \partial N)_{A, T}$ $\phi=-(\partial F / \partial A)_{N, T}$, where $F$ is the Helmholtz free energy, $A$ is the area of the substrate and $N$ the number of atoms adsorbed.

Price and Venables [17] constructed a model of the solid phases based on the Bragg-Williams partition function

$$
Q=\frac{M !}{N !} \frac{q^{N}}{(M-N) !} \exp \left(\frac{-N^{2} \psi}{2 M k T}-\frac{N P}{k T}\right),
$$

where the $N$ atoms are on $M$ sites, $P$ is the rare-gas-graphite potential, $\psi$ is the potential energy of an atom in the adlayer and $q$ is the internal partition function of each atom. They showed, by minimizing $F$ with respect to $\theta=N / M$, that the vacancy concentration $(1-\theta)$ was $<10^{-4}$ for reasonable potentials at the temperatures $(\leq 90 \mathrm{~K})$ of interest for solid monolayers. In this case the expression for $F$ simplifies to

$$
F / N k=\psi / 2+P-T \ln q .
$$

This expression can be used to evaluate $\mu$ and $\phi$ and hence the conditions for equilibrium lines and isosteres. However, in repeating the (PV) calculation, we have found it easier to evaluate $F$ directly, because $F / A$ is a minimum at equilibrium. We choose a reference state which is the localized solid phase with $n=N / A=1$ and express $F / A$ as $F_{1}$ (per graphite site) in $\mathrm{K}$ units. Then the localised solid has

$$
F_{1}=\psi_{1} / 2+P_{0}-\delta-T \ln q_{1},
$$


where, in the (PV) model, localization is favoured by a potential energy $\delta$ below the average energy $P_{0}$ because all the adatoms sit in the graphite potential wells.

The unlocalised solid $(n \neq 1)$ has a corresponding $F_{2}$ given by

$$
F_{2}=(1-n) \mu_{\mathrm{g}}+n\left(\psi_{2} / 2+P_{\mathrm{o}}-T \ln q_{2}\right),
$$

where the first term accounts for the adatoms which have left the surface for the vapour (or vice versa). The equilibrium adsorbed structure : localised (in registry) solid, unlocalised (out of registry) solid or low density adsorbed gas is then simply given by whichever of $F_{1}, F_{2}$ or $\mu_{\mathrm{g}}$ is lowest. In the case of the unlocalised solid the density $n$ is that which minimises $F_{2}$; for the adsorbed gas a full calculation retaining $n$ in equation (9) is straight-forward, but for practical purposes $n=0$, and hence $F \rightarrow \mu_{\mathrm{g}}$ is quite adequate. The above formulae are equivalent to the (PV) formulae for $\theta=1$.

3.2 INTERATOMIC POTENTIALS AND LATTICE DYNAMICS. - The model depends principally on the interatomic potentials used to determine $P_{0}, \delta$ and $\psi$, and also at finite temperatures on the form of the partition function $q$, i.e. on the dynamical model adopted. Here we follow (PV) closely. The average potential $\boldsymbol{P}_{0}$ seen by the gas atom isolated on the graphite can be determined by calculation using Lennard-Jones (LJ) potentials $[20,21]$ or more reliably by comparison with Henry law adsorption isotherms at low coverage. The values are $-1410 \mathrm{~K}$ for $\mathrm{Kr}$ and $-1858 \mathrm{~K}$ for $\mathrm{Xe}$ [17]. The extra energy which the rare gas atom feels when it is at the bottom of the potential well in the graphite, $\delta$, is expressible in terms of the values of $P$ at different points on the graphite lattice, and has values estimated by $\mathrm{LJ}$ potential calculations as $\delta=25,27 \mathrm{~K}[17,20]$, $\delta=28,25 \mathrm{~K}[21]$ for $\mathrm{Xe}$ and $\mathrm{Kr}$ respectively. While these values do not necessarily determine $\delta$ absolutely, they do indicate that $\delta$ is small and that the graphite surface is smooth enough for us to neglect the variation in height of a rare gas atom with position on the graphite surface.

Recently new multi-parameter potential functions (MP) for pairs of rare-gas atoms have been developed and these have essentially displaced the LJ potential for all but illustrative purposes (cf. reference [10] Chapter 4). Potentials for $\operatorname{Kr}[22,24]$ and for $\mathrm{Xe}[24,25]$ have been described. The various variants are negligibly different for our purposes and we have used the $\mathrm{K} 2$ potential for $\mathrm{Kr}$ and the $\mathrm{X} 2$ potential for $\mathrm{Xe}$ from reference [22]. With these potentials the long-range triple-dipole energy (the Axilrod-Teller-Muto (ATM) 3-body energy) is also used, and in $3 \mathrm{D}$ bulk rare gas solids this repulsive energy compensates for the greater depth of the pair potential with respect to the LJ potential.

In a $2 \mathrm{D}$ situation, this compensation is less complete, since the 3-body term is less important than in $3 \mathrm{D}$. However, there is also another repulsive energy on the graphite surface which is the analogue of the ATM energy, with the graphite as the third body. This energy is the Sinanoglu-Pitzer (SP) term $[25,17]$. There have been several discussions of the magnitude of the SP energy $[26,27,28,17]$ and it is of considerable interest to determine this from comparison with a realistic model of rare gas adsorption on graphite. This is discussed further in $\S 4.1$.

The lattice dynamics of both the substrate and the adsorbed layer will influence the thermodynamics of adsorption. In the present model we neglect the lattice vibrations of graphite because of its high Debye temperature. For the $\mathrm{Kr}$ and the $\mathrm{Xe}$ we use the Devonshire cell model (D) for the $x-y$ motion as we feel it describes the anharmonic effects of a classical atom remarkably well (cf. reference [10] chapter 6 ; 29) ; for the $z$-motion we use the Einstein model as (PV). We thus set out to compare the multiparameter potentials with the cell partition function (the MPD model) and various variants of the MPSPD model with the diffraction results described in $\S 2.2$. For comparison, we note that the lattice expansion of solid $3 \mathrm{D} \mathrm{Kr}$ and $\mathrm{Xe}$ is given to within $0.2 \%$ and $0.8 \%$ respectively in the range 45-90 K [30]. Thus we might expect the cell model, when we employ the right potential for the $2 \mathrm{D}$ situation, to be in error by $\$ 1.6 \%$ in the value of $n$ for $\mathrm{Xe}$ and probably better for $\mathrm{Kr}$.

3.3 THE SOLID-SOLID TRANSTTION, THE INFLUENCE OF MISFIT DISLOCATIONS. - In the (PV) model outlined above, the driving force for localization in the graphite potential wells is the extra potential energy gained, $\delta$, when $n=1$. For all $n \neq 1$ this energy is set $=0$. This result is indeed correct if we insist that the lattice parameter of the adsorbed layer is everywhere uniform. However the system can lower its energy by modulating the lattice parameter to take advantage of the potential wells, thus producing an array of misfit dislocations between the adsorbed layer and the graphite. These misfit dislocations are seen in many systems by electron microscopy, and have been seen in thicker Xe films on graphite [31].

The existence of misfit dislocation array explains the epitaxial order observed in diffraction experiments. The stability of this array arises because in the dislocation model the free energy gain for $n \neq 1$ is not zero except for large deviations from one, i.e. for large misfit, $|m|$. In particular, the gain is of form $\delta f(m)$ where $f(m) \simeq 1-A|m|$ as $m \rightarrow 0$ and $f(m) \rightarrow 0$ as $|m|$ becomes large. Here $A$ is a constant proportional to the dislocation energy, which may, however, be difficult to calculate accurately.

In an adsorbed layer, even if solid, the 
dislocations cannot be stationary, since atoms are interchanging with the vapour, and the most likely place for attachment is onto free ends in the dislocation network. This is almost certainly also the way in which the lattice parameter, and hence the spacing of the dislocation array, accommodates itself to changes in $p$ and $T$. If this motion is important in contributing to the free energy of the array then the layer will be further stabilised. This unsolved problem has much in common with the dynamics of solitons [32].

If the energy of dislocations (i.e. $A$ ) is small enough, the solid-solid phase transition in $\mathrm{Kr}$ and Xe monolayers can be second order, as the experimental results of figures 3 and 4 indicate. This is illustrated schematically in figure 6 for the case of $X e$, and $m \geq 0$. The free energy $F=F_{2}-F_{1}$ in equations (8) and (9) is shown in figure $6 \mathrm{a}$. At low $T\left(<T_{1}\right), \quad F_{1}<F_{2}$ and the localised phase is favoured due to $\delta$. At $T=T_{1}$, a first order transition is predicted from $m=0$ to $m=m_{0}\left(T_{1}\right)$, and at $T>T$, the unlocalised phase with $m=m_{0}(T)$ is stable. This is the (PV) model.

The dislocation contribution is sketched in figure $6 \mathrm{~b}$, (i) for the static energy and (ii) if dynamic effects are important. When the two contributions (a) and (b) are combined we get figure $6 c$. If $A$ is large we get curve (iii) which predicts a first order

(a)

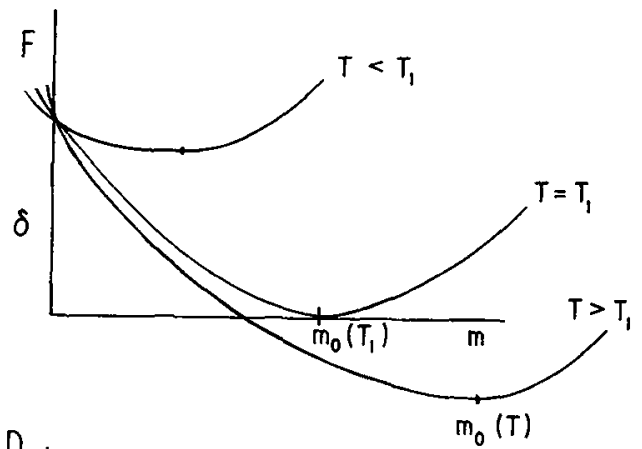

(b)

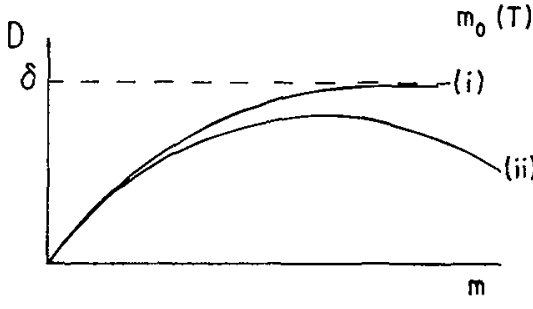

(c)

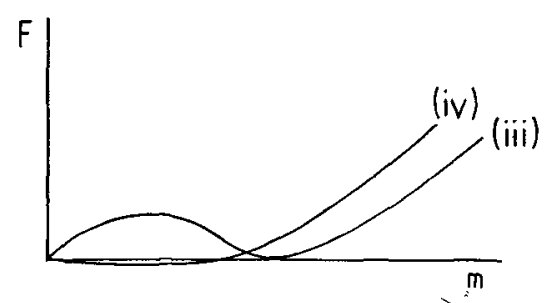

Fig. 6. - Schematic free energy diagram for $\mathrm{Xe}$ on graphite $\left(m \geq 0\right.$ ). See text for discussion. (a) $F=F_{2}-F_{1}$ for different temperatures; (b) Dislocation contributions (i) and (ii); (c) Composite curves for (iii) first order and (iv) second order transition. transition with somewhat reduced $T_{1}$ and $m_{0}\left(T_{1}\right)$. If however, $A$ is small enough so that the slope becomes zero at $m=0$ before the first order transition occurs then curve (iv) results. This is a second order transition with $m(T)$ increasing linearly with $T$ initially for $T>T_{1}$. This behaviour is discussed in more detail elsewhere [33] but it is clear that it mirrors the essentials of the experimental situation for $\mathrm{Kr}$ and $\mathrm{Xe}$. Results of model calculations are given in $\$ 4.2$.

The above approach is equivalent to a mean field model of the dislocations, and gives a second-order transition in the way sometimes referred to as Landau theory [36]. We have not explored other approaches to second order phase transitions e.g. [37].

4. Comparison of the models with diffraction experiments. - 4.1 NON-DISLOCATED MODELS. The isosteres for the unlocalised phase for the MPD and MPSPD models have been given by Price and Venables [17]. In repeating their calculations we have found that the ATM energy was somewhat overestimated (by about $10 \mathrm{~K}$ only in $\psi / 2$ ), with the result that their values of $n$ should be decreased by about 0.02 for $\mathrm{Kr}$ and 0.01 for Xe. Corrected $\log p-1 / T$ plots are given in figures 6 and 7 for $\mathrm{Kr}$ and $\mathrm{Xe}$, with an extended range to allow comparison with the newer diffraction data. Also added is the predicted gas-solid transition line. The two models shown are the MPD and the MP0.4SPD (with the SP term at $40 \%$ strength) which we think is close to reality.

From the expected values of $\delta$ it is-clear that $\mathrm{Kr}$ condenses into the localised $(n=1)$ phase, whereas Xe condenses into a non-localised phase with $n<1$, as discussed by PV. Because the gas free energy $\mu_{\mathrm{g}}$ varies so rapidly with $T$ and $p$, the gas-solid transition line is rather insensitive to fine details of the solid free energy. For both $\mathrm{Kr}$ and $\mathrm{Xe}$ the MPD and the MP0.4SPD models bracket the experimental line. If one suggests that the experimental line [6] should pass through the experimental triple point, then this indicates that the MP0.4SPD is more realistic. This conclusion is reinforced when dislocations are included as these stabilise the solid by a small amount and depress the theoretical transition line. In any case the MP0.4SPD model is well within experimental error and the MPD model is also very close.

The value of $n$ for $\mathrm{Xe}$ at the gas-solid transition $\left(=0.89\right.$ for $\left.10^{-6}<p<5 \times 10^{-8}\right)$ also favours the MP0.4SPD model. From the diffraction experiments (Fig. 3) $m=0.06 \pm 0.003$ and hence $n=0.890 \pm 0.006$ in this pressure range.

On the model of PV the value of $\delta$ can be deduced from the position of the solid-solid transition. Using the new calculations to compare with the diffraction data, we find that the values for the MP0.4SPD 

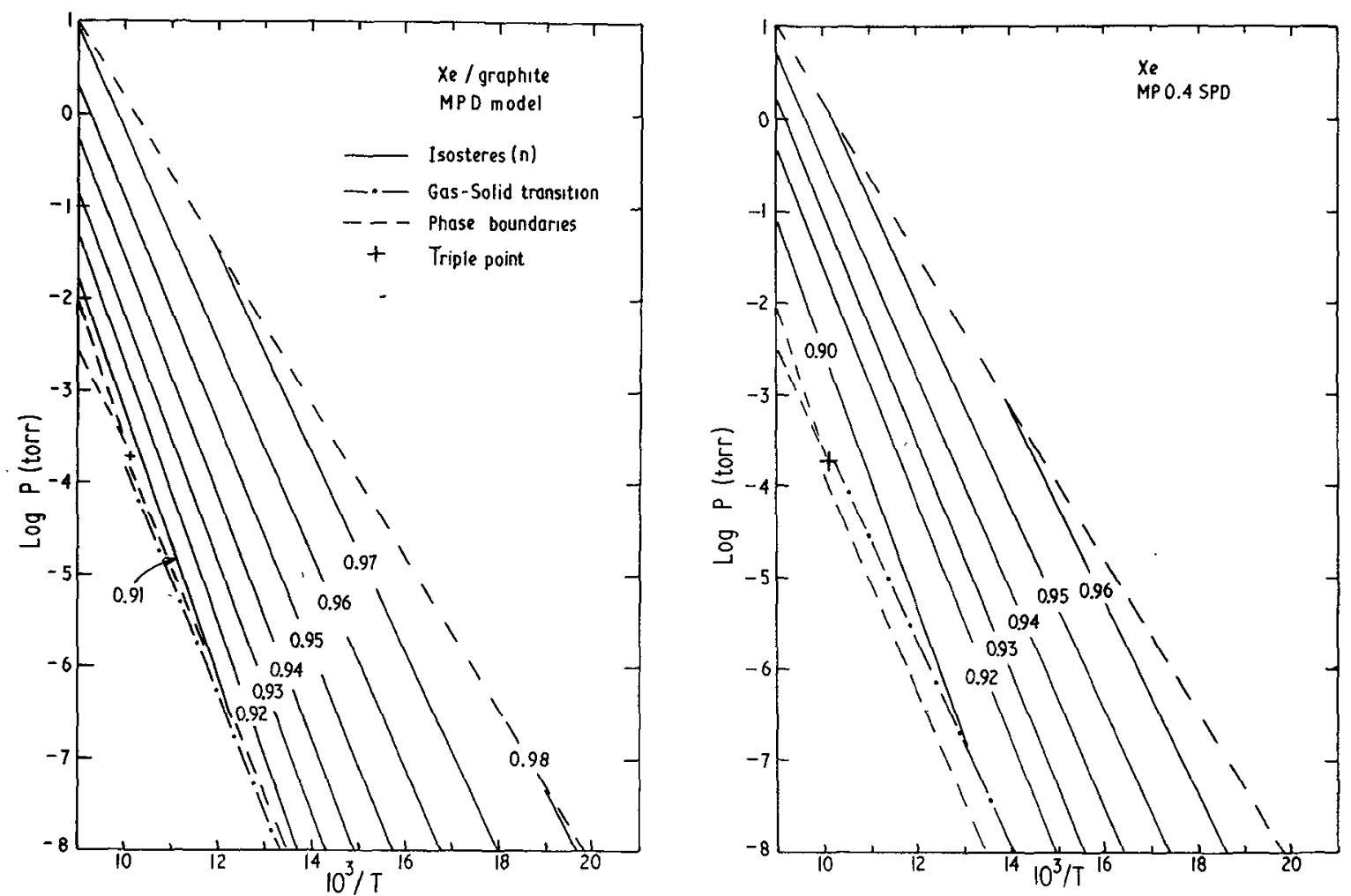

FIG. 7. - Log p versus $1 / T$ plots for Xe on the $(a)$ MPD and $(b)$ MP0.4SPD models, showing calculated isosteres and experimental data from figure 1.
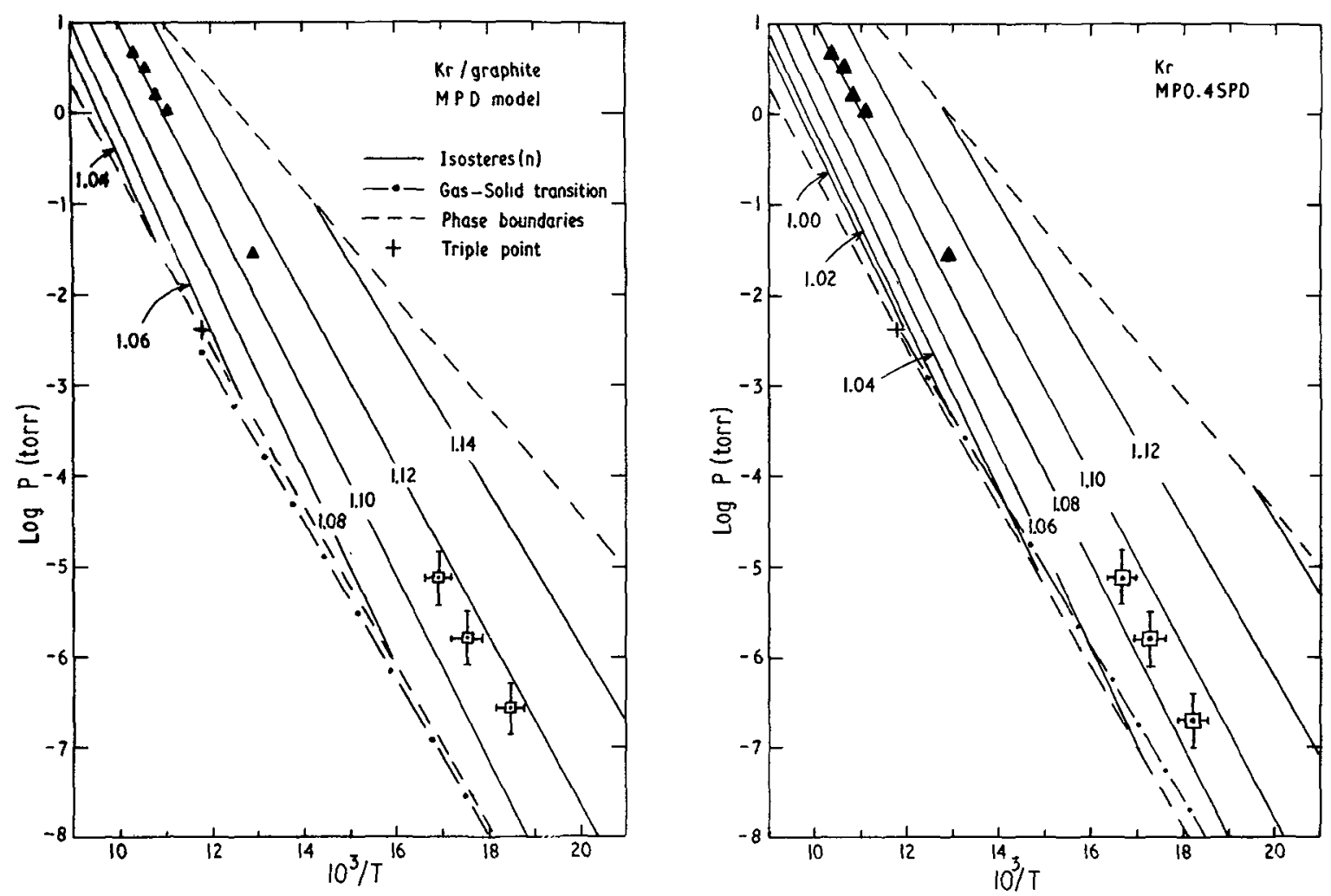

FiG. 8. - $\log p$ versus $1 / T$ plots for $\mathrm{Kr}$ on the $(a)$ MPD and $(b)$ MP0.4SPD models, showing calculated isosteres and experimental data from figure 2 
(MPD) models would be $24.5 \pm 3(40.5 \pm 4.5)$ for $\mathrm{Kr}$ and $21.5 \pm 2(10.0 \pm 1.5)$ for $\mathrm{Xe}$. The values for the MP0.4SPD model are quite close to the values calculated from Lennard-Jones potential $[20,21]$.

However, as we have noted, the form of the predicted transition is first order, not in agreement with experiment, and for this reason we have examined dislocation models which can give a second order transition.

4.2 Dislocated MODELS. - Dislocations can be inserted into the PV model by letting the energy gain from orientational epitaxy be of the form $\delta . f(m)$ as explained in \$3.3. At a fundamental level the problem is now much more complex, since the energies of hexagonal arrays of dislocations are involved. Indeed even dislocation interactions are essential as these determine the form of $f(m)$ and hence the predicted $m$ value. Moreover there is at least one more parameter involved for any particular form of $f(m)$; this is $f^{\prime}(0)=-A$. For a given model and value of $\delta$, the solid-solid transition point depends also on $A$. Hence finding a unique solution is not really possible. What we can do at this stage is to show that particular dislocation models can give reasonably good agreement with the experimental results for a suitable choice of parameters. It is left to future work to see whether these parameters agree in detail with $2 \mathrm{D}$ (hexagonal) dislocation models.

In general the introduction of the dislocations pushes the predicted transition point to the left on figures 3 and 4 . To compensate for this and bring back the transition point to agree with experiment, we have, within a given model, to increase $\delta$. If we have the transition in the right place the parameters $\delta$ and $A$ have to be related, i.e. $\delta$ increases as $A$ decreases. But there is still no assurance that the chosen form of $f(m)$ will reproducce the misfit curve $m(T, \log p)$. A first-order or second-order transition, or a combination, can be predicted, depending on the details of $f(m)$, indeed of $f^{\prime}(m)$ [33]. We have studied three forms of $f(m)$ in order to explore this problem. These are the exponential $f(m)=\mathrm{e}^{-A|m|}$ : piece-wise linear, $f(m)=1-A|m|$ for $A|m| \leq 1,0$ otherwise : and an adaptation of the linear dislocation model of Frank and Van der Merwe [34]. In this case the form of $f(m)$ is detailed elsewhere [33], but for small $m$ it is more linear than exponential and then goes to zero more slowly.

The exponential form gives a curve which usually increases too steeply at small $m$ and too slowly at high $m$ : i.e., the shape of the curve is nearer to a first order transition than experiment. The piece-wise linear curve starts too slowly and then jumps in a first order transition. The Frank-Van der Merwe form, which contains the correct physics of dislocations in one-dimension for a harmonic lattice, can be made to reproduce the form of $m$ quite closely. However, we are convinced that anharmonic effects are important, and that further development of dislocation models is needed. The best fits we have obtained with the exponential and linear Frank-Van der Merwe dislocation models are shown in figure 9 for $\mathrm{Xe}$ and figure 10 for $\mathrm{Kr}$ [33].

The absolute positions of the transitions are fairly sensitive to the assumed model and to the accuracy with which the undislocated lattice parameter is known for the model. Typically a shift in the fraction

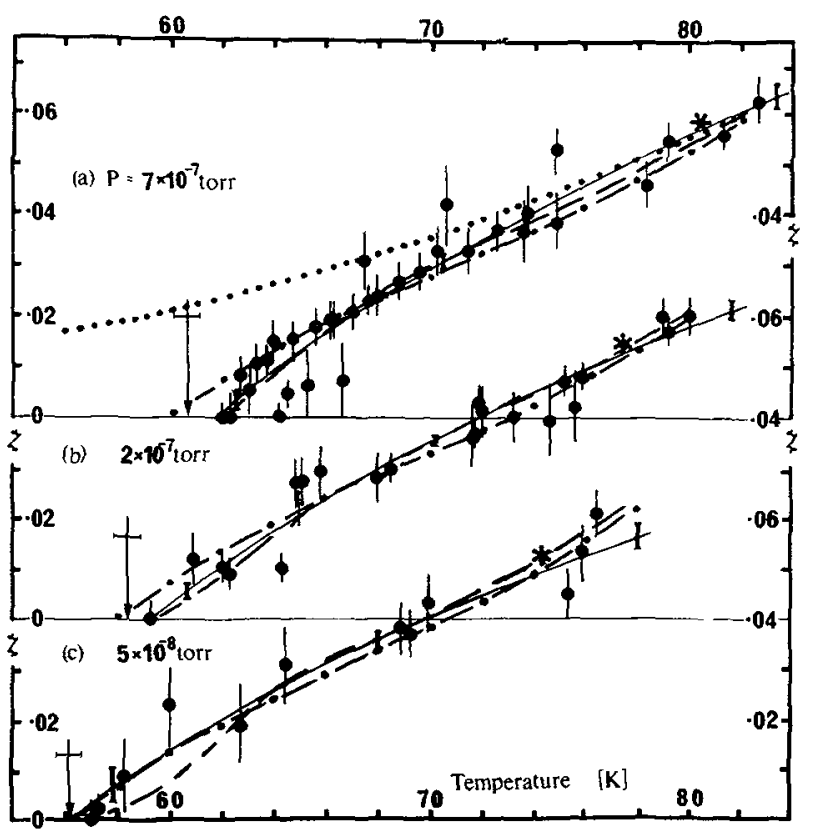

FIG. 9. - Best -fit curves for MP0.4SPD model with dislocations to Xe lattice parameter data. Curves : Full line, experimental least squares fit [16]; Dashed line, 1D-Frank-Van der Merwe model $[33,34]$ with $A=55, \delta=33$; dot dashed line, exponential model with $A=55, \delta=33$; dotted line, undislocated model for $p=7 \times 10^{-7}$ torr (Fig. $7 b$ ) Stars indicate the positions of the predicted gas-solid transition.

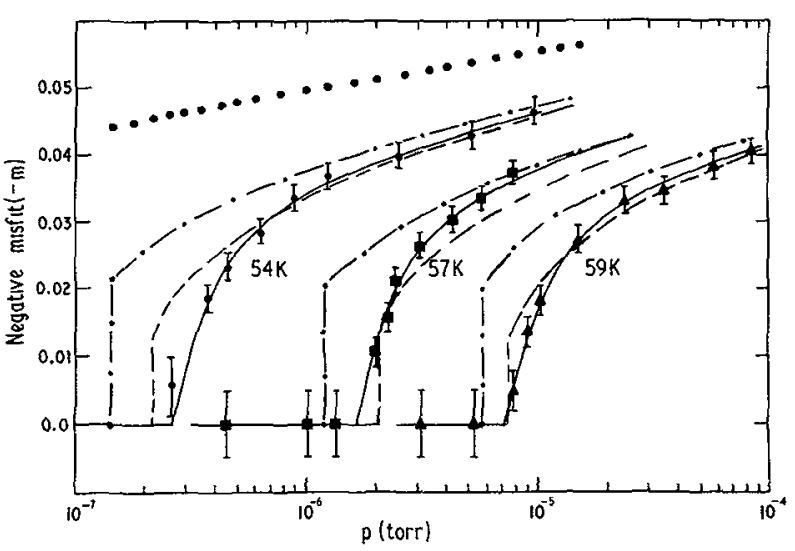

FIG. 10. - Best-fit curves for MP0.4SPD model with dislocations to $\mathrm{Kr}$ lattice parameter data. Curves: Full line, experimental [15]; Dashed-line, $1 \mathrm{D}$ Frank-Van der Merwe model $[33,34]$ with $A=19.2, \delta=50$; dot-dashed line, exponential model with $A=19.2, \delta=50$; dotted line, undislocated model for $54 \mathrm{~K}$ only (Fig. $8 b$ ). 
of the SP term by 0.2 will change $m$ by 0.005 and the transition pressure by a factor of five. Bearing the accuracy of the cell model and other parameters in mind, we feel that the best model has a SP term in it, with a strength of $0.4 \pm 0.2$ of that originally proposed, for both $\mathrm{Kr}$ and $\mathrm{Xe}$. This is close to the value found from second virial coefficient measurements by Wolfe and Sams [35].

4.3 DIFFRACTION INTENSITIES AND MONOLAYER STRUCTURE. - We argued in $\$ 2.2$ that misfit dislocations will also affect intensities of diffraction, since distortions give rise to destructive interference. We noted that the THEED intensities were only $0.29 \pm 0.03$ and $0.22 \pm 0.02$ of that expected (for the (10) and (20) spots respectively) for the undislocated monolayer in a particular experiment on Xe.

We simply note here that these values are of the order of magnitude expected for the simplest model of a hexagonal network of dislocations, shown in figure 11. [33]. This figure can be viewed at two

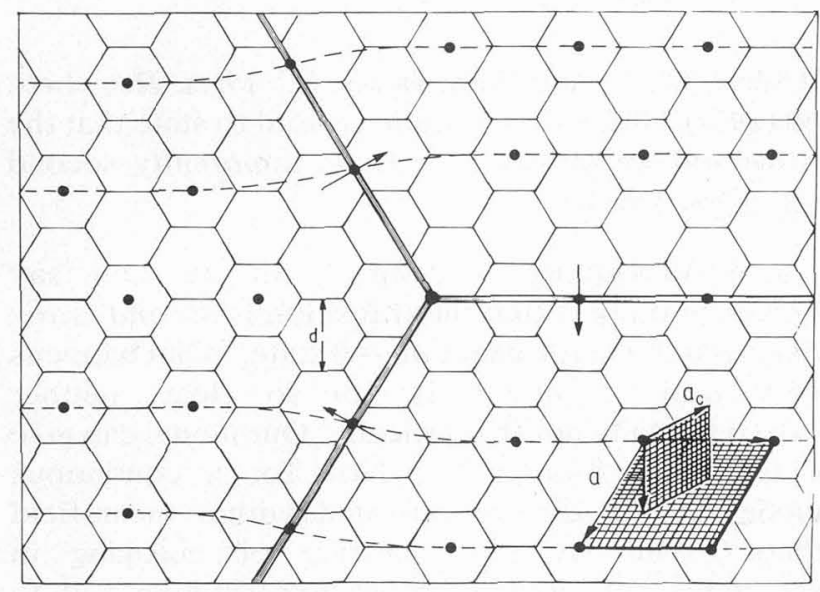

FIG. 11. - Visualization of dislocation structure, drawn for $\mathrm{Xe}(m>0)$. See text for discussion

scales. At the scale of the dislocation network, the hexagons represent the (edge) dislocations with spacing $d$, inversely proportional to $m$. The heavy lines represent a node of the network at the scale of the graphite hexagons, and the dots represent the xenon atoms in the dislocated monolayer. The dislocations have Burgers vectors b (indicated by arrows) of magnitude equal to graphite spacing $a_{\mathrm{c}}$. The (10) planes of the xenon are shown dashed.

Because a given (10) plane traverses 2 types of domain displaced relatively by a third of a lattice spacing, simple kinematic diffraction theory shows that, for narrow dislocations (small $l_{0}$ in the linear dislocation model [34]) the diffraction intensity in both (10) and (20) spots will be 0.25 of the undistorted intensity. For finite $l_{0}$, i.e. $l_{0}$ a sizeable fraction of $d$, the (10) intensity will increase and the $(20)$ decrease, in agreement with the preliminary experimental findings. Thus a detailed study of diffraction intensities may also produce additional evidence for the influence of dislocations.

5. Conclusions. - By studying detailed models of solid adsorbed $\mathrm{Kr}$ and $\mathrm{Xe}$ on graphite, we have shown that :

(i) the Sinanoglu-Pitzer term in the interaction between rare gas atoms on a substrate is around 0.4 of the strength originally proposed [25]. By considering the accuracy of the cell model we estimate this value to be $0.4 \pm 0.2$. We note that this is very similar to the values obtained from second virial coefficient measurements of Ar by Wolfe and Sams [35] ;

(ii) dislocations influence the form of the solid-solid transition and can cause it to be of second order ; a dislocation energy in the form given by Frank and Van der Merwe [34] can produce reasonable agreement with lattice parameters obtained from diffraction experiments ;

(iii) dislocations also influence the intensity of diffraction from a solid monolayer; preliminary experiments can be understood in terms of the simplest type of hexagonal dislocation network.

Acknowledgments. - We acknowledge initial help on the dislocation calculations by Dr. H. M. Kramer ; correspondence and preprints from Pr. S. C. Fain, Jr. ; Dr. A. Penney and the Royal Greenwich Observatory for the use of the computer controlled densitometer, and financial support from the Science Research Council, the British Council and CONACYT (Consejo Nacional de Ciencia y Tecnologia, Mexico).

\section{References}

[1] Dash, J.G., Films on Solid Surfaces (Academic Press) 1975. [2] TномY, A. and Duyal, X., J. Chim. Phys. 66 (1969) 1966; 67 (1970) 286, 1101.

[3] Thomy, A., ReGnier, J. and Duval, X., Colloques Internationaux $d u$ CNRS 201 (1973) $511 ; J$. Cryst. Growth 13/14 (1972) 159.

[4] LAHRER, Y., J. Chem. Soc. Faraday Trans I 70 (1974) 320.

[5] Putnam, F.A. and ForT, J. Jr., J. Phys. Chem. 79 (1975) 459.

[6] Suzanne, J., Coulomb, J.P. and Bienfait, M., Surf. Sci. 40 (1973) $414 ; 44$ (1974) 141.
[7] Kramer, H.M. and SuZAnne, J., Surf. Sci. 54 (1976) 659. [8] Bienfart, M. and Venables, J.A., Surf. Sci. 64 (1977), in press.

[9] QUENTEL, G., RICKARD, J.M. and KERN, R., Vide 164 (1973) 65 ; Surf. Sci. 50 (1975) 343.

[10] M.L. Klein and J.A. Venables eds. Rare Gas Solids I (Academic Press) 1976.

[11] Mutaftschiev, B., Surf. Sci. 61 (1976) 93.

[12] KJems, J.K., Passell, L., Taub, H., Dash, J.G. and Novaco, A.D., Phys. Rev. B 13 (1976) 1446. 
[13] McTague, J.P. and Nielsen, M., Phys. Rev. Lett. 37 (1976) 596.

[14] LANDER, J.J. and MORRISON, J., Surf. Sci. 6 (1967) 1.

[15] Chinn, M.D. and Fain, S.C. Jr., J. Vac. Sci. Technol. 14 (1977) 314 ; to be published.

[16] Venables, J.A., Kramer, H.M. and Price, G.L., Surf. Sci. 55 (1976) $373 ; 57$ (1976) 782.

[17] PRICE, G.L. and Venables, J.A., Surf. Sci. 59 (1976) 509.

[18] Hrll, T.L., J. Chem. Phys. 17 (1949) 520.

[19] LÀHRER, Y., Thèse, Faculté des Sciences d'Orsay (1970).

[20] Price, G.L., Surf. Sci. 49 (1975) 264.

[21] Steele, W.A., Surf. Sci. 36 (1973) 317.

[22] Barker, J.A., WaTtS, R.O., LeE, J.K., Schafer, T.P. and LEE, Y.T., J. Chem. Phys. 61 (1974) 3081.

[23] BARKer, J.A., KLein, M.L. and BobetiC, M.V., I.B.M. J. Res. Dev. 20 (1976) 222.

[24] Gough, D.W., Smith, E.B. and Maitland, G.C., Mol. Phys. 27 (1974) 867.

[25] Sinanoglu, O. and Pitzer, K.S., J. Chem. Phys. 32 (1960) 1279.
[26] Sams, J.R., Constabaris, G. and Halsey, G.D., J. Phys. Chem. 64 (1960) 1689 ; J. Chem. Phys. 36 (1962) 1334.

[27] Johnson, J.D. and Klein, M.L., Trans. Faraday Soc. 60 (1964) 1964.

[28] EvereTt, D.H., Discuss. Faraday Soc. 40 (1965) 177.

[29] Westera, K. and Cowley, E.R., Phys. Rev. B 11 (1975) 4008.

[30] BRICHENO, T. and VENABlES, J.A., unpublished work.

[31] Kramer, H.M., J. Cryst. Growth 33 (1976) 65.

[32] Varma, C.M., Phys. Rev. B 14 (1976) 244.

[33] Venables, J.A. and Schabes-Retchkiman, P.S., Surf. Sci. in press.

[34] Frank, F.C. and Van der Merwe, J.H., Proc. R. Soc. A 198 (1949) 205, 216.

[35] Wolfe, R. and SAMS, J.R. Jr., J. Chem. Phys. 44 (1966) 2181.

[36] Landau, L.D. and LifShitz, E.M., Statistical Physics (Pergamon Press) 1960, Chapter 14.

[37] Wilson, K.G., Rev. Mod. Phys. 47 (1975) 773.

[38] Marti, C., and Thorel, P., These Proceedings, J. Physique Colloq. 38 (1977) C4-26.

\section{DISCUSSION}

S. C. FAIN. - In your model for the dislocation structure (Fig. II), how does the intensity vary with misfit for the overlayer diffraction beams ?

J. A. VenABLEs. - This is a complex question, and the answer depends in detail on the atomic positions in the overlayer, and on the dislocation spacing in relation to the coherence width of the beam. We have not performed this calculation in any detail. The simple argument given here suggests that

$$
I_{\mathrm{g}} / I_{\mathrm{o}} \simeq 4 \%
$$

that for a perfect undislocated layer for various dislocations, for the reflections (ho), given a large coherence width, and this seems to be borne out by our preliminary experiments.

For wider dislocations, or for a more sinusoidal variation of the positions of the atoms, which is an equivalent statement, the reduction in intensity will be less marked. But the diffraction pattern should also contain satellite spots and the intensities in these spots will also change according to the details of the atomic positions. However, many of these satellite intensities may be below the detection limit experimentally. In all these cases the most abrupt changes should occur across the registry-disregistry transition. We hope to explore these effects by THEED in the future.

S. C. FAIN. - I would like to point out that Ying's more complete solution to the 1-D model of Frank and van der Merwe shows first-order transitions for strong substrate potentials [Ying, S. C., Phys. Rev. B12 (1971) 4160]. Our experimental data cannot rule out a discontinuity in lattice constant of less than $1 \%$ at the transition. Thus in presenting our data
[Chinn, M. D. and Fain, Jr., S. C., Phys. Rev. Lett. 39 (1977) 146], we were quite careful to state that the transition we observe for $\mathrm{Kr}$ is apparently second (or higher) order.

J. A. Venables. - Sure! All we can say experimentally is that the transition is second-order like away from the exact $m=0$ state. What happens very near to $m=0$ is not so clear, neither experimentally nor theoretically. Our model can give a true second-order transition for a continuous model of the dislocations and within mean-field theory. But we must beware of engaging in semantics and stick close to experiments and to simple explanations.

M. SCHICK. - Is it correct that you have built into your calculation, which employs dislocations, the second order transition?

J. A. Venables. - Not really. The calculation presented here, and in more detail in a companion paper to be published in Surface Science, is really a mean-field treatment of the effect of the dislocations. The model can produce a transition of first order, second-order or even both, depending on the details of the form of the dislocation energy, $\delta f(m)$. What we have shown is that with a suitable choice of the parameter $\delta$ and the form of $f(m)$, the second-order like behaviour of the lattice parameter of $\mathrm{Xe}$ and $\mathrm{Kr}$ can be simulated with some screens, and that the values of the parameters needed at physically reasonable. But the form of $m(T, p)$ predicted is quite sensitive to the form of the dislocation energy employed, as detailed in the companion paper. 